FORMATION Formation emploi

Revue française de sciences sociales

106 | avril-juin 2009

Pêle-mêle

\title{
Elargir le débat
}

Réponse à Philippe Trouvé

\section{Paul Santelmann}

\section{(2) OpenEdition}

Journals

Édition électronique

URL : http://journals.openedition.org/formationemploi/1960

DOI : 10.4000/formationemploi.1960

ISSN : 2107-0946

Éditeur

La Documentation française

Édition imprimée

Date de publication : 1 juin 2009

Pagination : 89-91

ISSN : 0759-6340

Référence électronique

Paul Santelmann, «Elargir le débat », Formation emploi [En ligne], 106 | avril-juin 2009, mis en ligne le 01 juin 2011, consulté le 30 octobre 2020. URL : http://journals.openedition.org/formationemploi/ 1960 ; DOl : https://doi.org/10.4000/formationemploi.1960

(c) Tous droits réservés 


\section{Élargir le débat Réponse à Philippe Trouvé}

Paul Santelmann*

\section{P. Santelmann répond au commentaire de P. Trouvé sur son article "Les distorsions diplômes/qualifications : l'exemple des techniciens et agents de maîtrise de type industriel ", paru dans le numéro 105 de Formation Emploi.}

Il est normal que Philippe Trouvé puisse estimer univoque ma contribution qui ne prétendait pas clore le débat mais plutôt l'ouvrir à nouveau dans une période charnière où les disjonctions évoquées de la relation emploi/formation vont prendre plus d'ampleur dans leurs effets induits. Le nombre d'éléments qui influencent la relation formation/emploi ne prédispose d'ailleurs pas à s'en tenir au format des articles de la revue Formation Emploi. Il me fallait donc choisir les points sur lesquels l'attention me semblait devoir être portée quant à l'appréciation des rôles respectifs de la formation initiale et de la formation des adultes mais aussi des comportements des entreprises.

Les arguments de Philippe Trouvé militent pour une appréciation plus nuancée et plus équilibrée des responsabilités respectives du système éducatif et des entreprises dans le décalage entre les logiques des filières diplômantes et les pratiques sélectives et organisationnelles des entreprises. À cet égard, mon propos peut paraître lapidaire et convenu mais interpelle un construit social qui n'a pas vocation à rester dans le marbre. Mes contributions antérieures ${ }^{1}$ sur les effets stigmatisants de la notion d'emploi non

1 «Formation professionnelle et emplois non qualifiés » Revue Droit Social $\mathrm{n}^{\circ} 4$ avril 2002 ; « Construction des diplômes professionnels et notion d'emploi non qualifié » in Méda D. et Vennat F. (2004), Le travail non qualifié, permanences et paradoxes, La Découverte.

* Paul Santelmann est chef du service prospective à la Direction générale de l'AFPA (Association nationale pour la formation professionnelle des adultes). Il est chargé d'enseignement à l'université de Paris I (La Sorbonne) et au CNAM (Conservatoire national des arts et métiers). II est notamment l'auteur de : La formation professionnelle continue, collection "Les études", La Documentation française, 2006 ; De l'efficacité en formation continue, Éditions Liaisons, collection « Entreprise \& Carrières », 2004 ; Qualification ou compétences, en finir avec la notion d'emplois non qualifiés, Éditions Liaisons, collection "Entreprise \& Carrières ", 2002 ; La formation professionnelle, nouveau droit de I'homme?, Éditions Gallimard, collection « Folio actuel, Le Monde », 2001. 
qualifié vont dans le même sens : ce qui a été stabilisé comme représentation de la relation emploi/ formation dans les années 50 et 60 nous a collectivement handicapés, au moment des " années chômage », dans la conduite des réformes du système de formation et dans l'instrumentation des marchés internes/ externes de l'emploi.

En ce qui concerne les responsabilités des uns et des autres, Philippe Trouvé considère que «je charge la barque» de l'Éducation nationale au détriment des entreprises. J'ai effectivement tendance à penser que, dans une démocratie, la responsabilité de l'État ne se situe pas exactement au même niveau que celles des entreprises. D'ailleurs, ce vocable «les entreprises» est malvenu dès lors que cet univers s'exprime par l'intermédiaire du dialogue social et du rôle joué par les partenaires sociaux. Par ailleurs, les comportements des entreprises sont un élément à prendre en compte dans la façon de conduire les systèmes éducatifs : faut-il les ignorer, s'y adapter mécaniquement ou tendre à les infléchir en favorisant les espaces de coopération de terrain permettant de corriger les hypothèses quelquefois abruptes qui sous-tendent tous les systèmes éducatifs ?

Quant à la conception des diplômes professionnels, chacun conviendra que les acteurs du système éducatif et les partenaires sociaux, contrairement à d'autres pays, ne se sont pas retrouvés autour d'une doctrine forte permettant d'établir une dynamique vertueuse entre ambitions éducatives et construction des qualifications professionnelles. Les PME (petites et moyennes entreprises), confrontées à des difficultés de recrutement depuis des lustres, peuventelles être désignées comme responsables de cet état de fait? Mon propos est donc, j'en conviens, rudimentaire, et concerne précisément les tensions du marché de l'emploi et les phénomènes d'illisibilité des compétences qui s'y manifestent. En l'occurrence, il peut se résumer à cette interrogation: comment se fait-il que l'analyse des emplois industriels 《intermédiaires » correspondant à l'agrégat statistique des TAM - techniciens et agents de maîtrise - (effectivement hétérogène et effectivement illustratif d'une grille de lecture de plus en plus approximative des qualifications professionnelle) semble à la fois :
- Conforter l'hypothèse d'activités opérationnelles complexes mais relevant plus des nouvelles identités ouvrières que d'une variante amenuisée du métier d'ingénieur (et donc correspondant plutôt à des progressions professionnelles de type promotionnel des ouvriers qualifiés) ;

- Générer une batterie de BTS et de DUT (respectivement brevet de technicien supérieur et diplôme universitaire de technologie) alimentée essentiellement par les filières générales et technologiques initiales au lieu d'être, soit des diplômes de formation continue, soit l'aboutissement de l'enseignement professionnel dans le champ des techniques industrielles (ce qui interroge la pertinence du binôme $\mathrm{CAP} / \mathrm{Bac}$ pro quant à sa cohérence avec le niveau III - BTS-DUT ou fin de premier cycle de l'enseignement supérieur).

Philippe Trouvé, avec raison, souligne que la « surproduction » de diplômés en BTS et en DUT n'a pas entravé les mécanismes promotionnels. Outre qu'il est difficile d'évaluer finement ce que recouvrent ces mécanismes promotionnels, notamment en intégrant les effets des déclassements en début de carrière et les paramètres générationnels, de taille d'entreprise et de mobilités, ces processus de progression professionnelle d'ouvriers qualifiés mais peu diplômés ont-ils influencé ou modifié la conception des diplômes et la répartition de l'effort de formation initiale et continue? Il n'est pas anodin de soulever la question de l'efficience d'un système de formation qui concentre son effort avant 25 ans, démultiplie les filières et les niveaux et fait $\mathrm{du}$ déclassement en début de carrière l'aiguillon de la poursuite des études. Tout cela a un coût qui fait de notre enseignement professionnel (dans toutes ses composantes) le moins efficient de tous les pays développés (en termes de rapport coût/efficacité) et surtout le moins contributif à la formation promotionnelle en mi-carrière ! À peine quelques dizaines de milliers d'adultes accèdent, après l'âge de trente ans, à des formations qualifiantes de niveau IV (baccalauréat ou brevet technique) ou III! Par ailleurs, un nombre important de jeunes « stoppés " $\mathrm{au}$ niveau $\mathrm{V}$ ne parviennent plus à accéder à l'emploi, alors même qu'ils sont potentiellement capables d'exercer des emplois de base et même d'évoluer en cours de carrière sur ces fameux 
emplois de techniciens ciblés artificiellement par les BTS et DUT, diplômes inatteignables pour ces publics...

Ce qui est soulevé en contrepoint par cette situation renvoie :

- Aux profils des enseignants professionnels du secondaire et des enseignants des IUT (Institut universitaire de technologie) ;

- Aux caractéristiques des collectifs pédagogiques de la formation professionnelle initiale quant à leur capacité à articuler savoirs généraux, technologies et savoirs techniques/savoir-faire ;

- Au bilan de l'apprentissage et de l'alternance sous l'angle des apports formatifs respectifs des opérateurs de formation et des entreprises ;

- À la synergie entre la formation initiale et les opérateurs de formation des adultes comme l'AFPA (Association nationale pour la formation professionnelle des adultes).
La propension à multiplier les formules de formation spécialisées (la France possède cinq systèmes professionnels accessibles aux jeunes: enseignement professionnel scolaire, apprentissage, contrats de professionnalisation, AFPA et programmes Jeunes des régions) résulte bien d'une insuffisante réflexion sur les fondamentaux technico-professionnels à construire en formation initiale et sur les ressources formatives qu'il est nécessaire de développer et de consolider dans les entreprises. Les rappels et les éclairages de Philippe Trouvé n'infèrent d'ailleurs pas cette exigence mais démontrent qu'il faut parvenir à mieux objectiver les décalages de la relation emploi/ formation pour peser sur les réformes de notre système de formation. La résistance à l'égard du bac pro en trois ans témoigne, à cet égard, d'un débat insuffisant sur ce qu'il y a à refonder comme socle d'enseignement technologique et professionnel de base et comme articulation entre système éducatif et structure des qualifications professionnelles. 